\title{
A CURSE-OF-DIMENSIONALITY-FREE NUMERICAL METHOD FOR A CLASS OF HJB PDE'S
}

\author{
William M. McEneaney ${ }^{*, 1}$ \\ * Dept. of Mech. and Aero. Eng. and Dept. of Math., \\ University of California San Diego,wmceneaney@ucsd.edu
}

\begin{abstract}
Max-plus methods have been explored for solution of first-order, nonlinear Hamilton-Jacobi-Bellman partial differential equations (HJB PDEs) and corresponding nonlinear control problems. These methods exploit the max-plus linearity of the associated semigroups. Although these methods provide advantages, they still suffer from the curse-of-dimensionality. Here we consider HJB PDEs where the Hamiltonian takes the form of a (pointwise) maximum of linear/quadratic forms. We obtain a numerical method not subject to the curse-of-dimensionality. The method is based on construction of the dual-space semigroup corresponding to the HJB PDE. This dual-space semigroup is constructed from the dual-space semigroups corresponding to the constituent Hamiltonians. Copyright 2005 IFAC.
\end{abstract}

Keywords: partial differential equations, curse-of-dimensionality, dynamic programming, max-plus algebra, Hamilton-Jacobi-Bellman equations

\section{INTRODUCTION}

One approach to nonlinear control is through Dynamic Programming (DP). With DP, solution of the control problem "reduces" to solution of the corresponding partial differential equation (PDE). In the case of Deterministic Optimal Control or Deterministic Games (such as $\mathrm{H}_{\infty}$ control) where one player's feedback is prespecified, the $\mathrm{PDE}$ is a Hamilton-Jacobi-Bellman (HJB) PDE. The difficulty is that one must solve the HJB PDE.

Various approaches have been taken to solution of the HJB PDE. The most common methods by far all fall into the class of finite element methods (cf. (Bardi and Capuzzo-Dolcetta, 1997), (Dupuis and Boué, 1999), among many others). These require that one generate a grid over some bounded region of the state-space. Suppose the region over which

\footnotetext{
1 Research supported by NSF grant DMS-0307229. The author also thanks Prof. J. William Helton for helpful discussions.
}

one constructs the grid is rectangular. Suppose one uses 100 grid points per dimension. If the state dimension is $n$, then one has $100^{n}$ grid points. Thus the computations grow exponentially in state-space dimension $n$.

In recent years, an entirely new class of numerical methods for HJB PDEs has emerged (c.f. (Fleming and McEneaney, 2000), (McEneaney, 2003), (McEneaney, 2004), (Akian, Gaubert and Lakhouat, 2004)). These methods exploit the max-plus linearity of the associated semigroup. They employ a max-plus basis function expansion of the solution, and the numerical methods obtain the coefficients in the basis expansion. Much of the work has concentrated on the (harder) steady-state HJB PDE class. With the max-plus methods, the number of basis functions required still typically grows exponentially with space dimension. For instance, one might use 25 basis functions per space dimension to cover a rectangular region well. Consequently, one still has the 
curse-of-dimensionality. Even with the max-plus approach, one cannot expect to solve problems of more than say dimension 4 or 5 on current machinery.

This paper discusses an approach to certain nonlinear HJB PDEs which is not subject to the curse-of-dimensionality. In fact, the computational growth in state-space dimension is on the order of $n^{3}$. However, there is exponential computational growth in a certain measure of complexity of the Hamiltonian. Under this measure, the minimal complexity Hamiltonian is the linear/quadratic Hamiltonian - corresponding to solution by a Riccati equation. If the Hamiltonian is given as a maximum of $M$ linear/quadratic Hamiltonians, then one could say the complexity of the Hamiltonian is $M$.

The approach has been applied on some simple nonlinear problems. A few simple examples comprised of 3 linear/quadratic components were solved in 10-20 seconds over $\mathbf{R}^{3}$ and 10-45 seconds over $\mathbf{R}^{4}$. For these particular problems, the solution was obtained over the entire space with the resulting errors in the gradients growing linearly in $|x|$. (See Section 5 for specific examples.) These speeds are of course unprecedented. This code was not optimized. Further, the computational growth in going from $n=4$ up to say $n=6$ would be on the order of $6^{3} / 4^{3} \simeq 4$ as opposed to say more than $10^{4}$ for a finite element method.

We will consider HJB PDEs given as

$$
0=\widetilde{H}(x, \nabla V)=\max _{m \in\{1,2, \ldots, M\}}\left\{H^{m}(x, \nabla V)\right\}
$$

with boundary data $V(0)=0$ ( $V$ being zero at the origin). In order to make the problem tractable, we will concentrate on a single class of HJB PDEs of form (1). However, the theory can obviously be expanded to a much larger class.

\section{REVIEW OF THEORY}

Note that due to space limitations, the proofs of the results cannot be included here.

As indicated above, we suppose the individual $H^{m}$ are linear/quadratic Hamiltonians. Consequently, consider a finite set of linear systems

$$
\dot{\xi}^{m}=A^{m} \xi^{m}+\sigma^{m} w, \quad \xi_{0}^{m}=x \in \mathbb{R}^{n} .
$$

Let $w \in \mathcal{W} \doteq L_{2}^{l o c}\left([0, \infty) ; \mathbb{R}^{m}\right)$. Let the cost functionals and value functions be

$$
\begin{aligned}
& J^{m}(x, T ; w) \doteq \int_{0}^{T} \frac{1}{2} \xi_{t}^{m} D^{m} \xi_{t}^{m}-\frac{\gamma^{2}}{2}\left|w_{t}\right|^{2} d t \\
& V^{m}(x)=\lim _{T \rightarrow \infty} \sup _{w \in \mathcal{W}} J^{m}(x, T ; w) .
\end{aligned}
$$

Obviously $J^{m}$ and $V^{m}$ require some assumptions in order to guarantee their existence.

Assume that there exists $c_{A} \in(0, \infty)$ such that $x^{T} A^{m} x \leq-c_{A}|x|^{2}$ for all $x \in \mathbb{R}^{n}$ and $m \in \mathcal{M}$. Assume that there exists $c_{\sigma}<\infty$ such that $\left|\sigma^{m}\right| \leq$ $c_{\sigma} \forall m \in \mathcal{M}$. Assume that all $D^{m}$ are positive definite, symmetric, and let $c_{D}$ be such that $x^{T} D^{m} x \leq c_{D}|x|^{2}$ for all $x \in \mathbb{R}^{n}$ and $m \in \mathcal{M}$. Lastly, assume that $\gamma^{2} / c_{\sigma}^{2}>c_{D} / c_{A}^{2}$.

These assumptions guarantee the existence of the $V^{m}$ as locally bounded functions which are zero at the origin (cf. (McEneaney, 1998)).

The corresponding HJB PDEs are

$$
\begin{aligned}
& 0=H^{m}(x, \nabla V) \\
& =\frac{1}{2} x^{T} D^{m} x+\left(A^{m} x\right)^{T} \nabla V+\frac{1}{2} \nabla V^{T} \Sigma^{m} \nabla V \\
& V(0)=0
\end{aligned}
$$

where $\Sigma^{m} \doteq \frac{1}{\gamma^{2}} \sigma^{m}\left(\sigma^{m}\right)^{T}$. Let $\mathcal{G}_{\delta}$ be the subset of $C\left(\mathbb{R}^{n}\right)$ such that $0 \leq V(x) \leq \frac{c_{A}(\gamma-\delta)^{2}}{c_{\sigma}^{2}}|x|^{2}$. For $m \in \mathcal{M}$, let $P^{m}$ satisfy the algebraic Riccati equations

$$
0=\left(A^{m}\right)^{T} P^{m}+P^{m} A^{m}+D^{m}+P^{m} \Sigma^{m} P^{m} .
$$

Theorem 2.1. Each value function (4) is the unique classical solution of its corresponding HJB $\operatorname{PDE}(5)$ in the class $\mathcal{G}_{\delta}$ for sufficiently small $\delta>0$. Further, $V^{m}(x)=\frac{1}{2} x^{T} P^{m} x$ where $P^{m}$ is the smallest symmetric, positive definite solution of (6) In particular, there exists symmetric, positive definite $\bar{C}$ such that $V^{m}(x)-\frac{1}{2} x^{T} \bar{C} x$ is convex for all $m \in \mathcal{M}$.

The method we will use to obtain these value functions/HJB PDE solutions will be through the associated semigroups. For each $m$ define the semigroup

$S_{T}^{m}[\phi] \doteq \sup _{w \in \mathcal{W}} \int_{0}^{T} \frac{1}{2}\left(\xi_{t}^{m}\right)^{T} D^{m} \xi_{t}^{m}-\frac{\gamma^{2}}{2}\left|w_{t}\right|^{2} d t+\phi\left(\xi_{T}^{m}\right)$

where $\xi^{m}$ satisfies (2). By (McEneaney, 1998), the domain of $S_{T}^{m}$ includes $\mathcal{G}_{\delta}$ for all $\delta>0$.

Theorem 2.2. Fix any $T>0$. Each value function, $V^{m}$, is the unique smooth solution of $V=S_{T}^{m}[V]$ in the class $\mathcal{G}_{\delta}$ for sufficiently small $\delta>0$. Further, given any $V \in \mathcal{G}_{\delta}, \lim _{T \rightarrow \infty} S_{T}^{m}[V](x)=V^{m}(x)$ (uniformly on compact sets).

Recall that the HJB PDE of interest is (1) with $H^{m}$ given by (5). The corresponding value function is 


$$
\begin{aligned}
& \widetilde{V}(x)=\sup _{w \in \mathcal{W}} \sup _{\mu \in \mathcal{D}_{\infty}} \widetilde{J}(x, w, \mu) \\
& \doteq \sup _{w \in \mathcal{W}} \sup _{\mu \in \mathcal{D}_{\infty}} \sup _{T<\infty} \int_{0}^{T} l^{\mu_{t}}\left(\xi_{t}\right)-\frac{\gamma^{2}}{2}\left|w_{t}\right|^{2} d t
\end{aligned}
$$

where

$$
\begin{aligned}
& l^{\mu_{t}}(x)=\frac{1}{2} x^{T} D^{\mu_{t}} x, \\
& \mathcal{D}_{\infty}=\{\mu:[0, \infty) \rightarrow \mathcal{M}: \text { measurable }\},
\end{aligned}
$$

and $\xi$ satisfies

$$
\dot{\xi}=A^{\mu_{t}} \xi+\sigma^{\mu_{t}} w_{t}, \quad \xi_{0}=x .
$$

Define the semigroup

$$
\widetilde{S}_{T}[\phi]=\sup _{w \in \mathcal{W}} \sup _{\mu \in \mathcal{D}_{T}} \int_{0}^{T} l^{\mu_{t}}\left(\xi_{t}\right)-\frac{\gamma^{2}}{2}\left|w_{t}\right|^{2} d t+\phi\left(\xi_{T}\right)
$$

where $\mathcal{D}_{T}=\{\mu:[0, T) \rightarrow \mathcal{M}:$ measurable $\}$.

Theorem 2.3. Fix any $T>0$. Value function $\widetilde{V}$ is the unique continuous solution of $V=\widetilde{S}_{T}[V]$ in the class $\mathcal{G}_{\delta}$ for sufficiently small $\delta>0$. Further, given any $V \in \mathcal{G}_{\delta}, \lim _{T \rightarrow \infty} \widetilde{S}_{T}[V](x)=\widetilde{V}(x)$ (uniformly on compact sets). Lastly, there exists $c_{V}>0$ such that $\tilde{V}(x)-\frac{1}{2} c_{V}|x|^{2}$ is convex.

\section{MAX-PLUS DUAL OPERATORS}

We use $\oplus, \otimes$ to indicate max-plus addition and multiplication; max-plus integration (supremization) is indicated by an $\oplus$ superscript on the integral sign. Let $\overline{\mathbb{R}}=\mathbb{R} \cup\{-\infty\}$. Recall that a function, $\phi: \mathbb{R}^{n} \rightarrow \overline{\mathbb{R}}$ is semiconvex if given any $R \in(0, \infty)$ there exists $\beta_{R} \in \mathbb{R}$ such that $\phi(x)+\frac{\beta_{R}}{2}|x|^{2}$ is convex over $\bar{B}_{R}(0)=\left\{x \in \mathbb{R}^{n}\right.$ : $|x| \leq R\}$. We say $\phi$ is uniformly semiconvex with constant $\beta$ if $\phi(x)+\frac{\beta}{2}|x|^{2}$ is convex over $\mathbb{R}^{n}$. Let $\mathcal{S}_{\beta}=\mathcal{S}_{\beta}\left(\mathbb{R}^{n}\right)$ be the set of functions mapping $\mathbb{R}^{n}$ into $\overline{\mathbb{R}}$ which are uniformly semiconvex with constant $\beta$. Note that $\mathcal{S}_{\beta}$ is a max-plus vector space (also known as a moduloid) (Fleming and McEneaney, 2000), (McEneaney, 2003), (Baccelli, Cohen, Olsder and Quadrat, 1992), (Cohen, Gaubert and Quadrat, 2004), (Litvinov, Maslov and Shpiz, 2001). Combining Theorems 2.1 and 2.3, we have

Theorem 3.1. There exists $\bar{\beta} \in \mathbb{R}$ such that given any $\beta>\bar{\beta}, \widetilde{V} \in \mathcal{S}_{\beta}$ and $V^{m} \in \mathcal{S}_{\beta}$ for all $m \in \mathcal{M}$. Further, one may take $\beta<0$ (i.e. $\widetilde{V}, V^{m}$ convex).

The following semiconvex duality result (Fleming and McEneaney, 2000), (McEneaney, 2003) requires only a small modification of convex duality and Legendre/Fenchel transform results (c.f. (Rockafellar and Wets, 1997)).
Theorem 3.2. Let $\phi \in \mathcal{S}_{\beta}$. Let $C$ be a symmetric matrix such that $C+\beta I>0$ (i.e. $C+\beta I$ positive definite) with either $C>0$ or $C<0$. Define $\psi: \mathbb{R}^{n} \times \mathbb{R}^{n} \rightarrow \mathbb{R}$ by $\psi(x, z)=-\frac{1}{2}(x-z)^{T} C(x-$ $z$ ). Then, for all $x \in \mathbb{R}^{n}$,

$$
\begin{aligned}
\phi(x) & =\max _{z \in \mathbb{R}^{n}}[\psi(x, z)+a(z)] \\
& \doteq \int_{\mathbb{R}^{n}}^{\oplus} \psi(x, z) \otimes a(z) d z \doteq \psi(x, \cdot) \odot a(\cdot)
\end{aligned}
$$

where for all $z \in \mathbb{R}^{n}$

$$
\begin{aligned}
& a(z)=-\int_{\mathbb{R}^{n}}^{\oplus} \psi(x, z) \otimes[-\phi(x)] d x \\
& =-\{\psi(\cdot, z) \odot[-\phi(\cdot)]\} \doteq\left\{\psi(\cdot, z) \odot\left[\phi^{-}(\cdot)\right]\right\}^{-} .
\end{aligned}
$$

We will refer to $a$ as the semiconvex dual of $\phi$.

Semiconcavity is the obvious analogue of semiconvexity. Let $\mathcal{S}_{\beta}^{-}$be the set of functions mapping $\mathbb{R}^{n}$ into $\mathbb{R} \cup\{+\infty\}$ which are uniformly semiconcave with constant $\beta\left(\phi(x)-(\beta / 2)|x|^{2}\right.$ concave over all of $\mathbb{R}^{n}$ ).

Lemma 3.3. Let $\phi \in \mathcal{S}_{\beta}$, and let $a$ be the semiconvex dual of $\phi$. Then $a \in \mathcal{S}_{\beta}^{-}$. Further, suppose $b \in \mathcal{S}_{\beta}^{-}$is such that $\phi=\psi(x, \cdot) \odot b(\cdot)$. Then $b=a$.

For simplicity, we will henceforth specialize to the case where $\psi(x, z) \doteq(c / 2)|x-z|^{2}$. It will be critical to the method that $\widetilde{S}_{\tau}[\psi(\cdot, z)] \in \mathcal{S}_{-(c+\varepsilon)}$ for some $\varepsilon>0$. This is the subject of the next theorem.

Theorem 3.4. We may choose $c>0$ such that $\widetilde{V}, V^{m} \in \mathcal{S}_{-c}$, and such that there exists $\bar{\tau}>0$ and $\eta>0$ such that ,

$\widetilde{S}_{\tau}[\psi(\cdot, z)], S_{\tau}^{m}[\psi(\cdot, z)] \in \mathcal{S}_{-(c+\eta \tau)} \quad \forall \tau \in[0, \bar{\tau}]$.

Henceforth, we suppose $c, \tau, \eta$ chosen so that the results of Theorem 3.4 hold. Now for each $z \in \mathbb{R}^{n}$, $\widetilde{S}_{\tau}[\psi(\cdot, z)] \in \mathcal{S}_{-(c+\eta \tau)}$. Therefore, by Theorem 3.2

$$
\widetilde{S}_{\tau}[\psi(\cdot, z)](x)=\psi(x, \cdot) \odot \widetilde{\mathcal{B}}_{\tau}(\cdot, z)
$$

where for all $y \in \mathbb{R}^{n}$

$$
\widetilde{\mathcal{B}}_{\tau}(y, z)=\left\{\psi(\cdot, y) \odot\left[\widetilde{S}_{\tau}[\psi(\cdot, z)](\cdot)\right]^{-}\right\}^{-}
$$

It is handy to define the max-plus linear operator with "kernel" $\widetilde{\mathcal{B}}_{\tau}$ as $\widehat{\widetilde{\mathcal{B}}}_{\tau}[a](z) \doteq \widetilde{\mathcal{B}}_{\tau}(z, \cdot) \odot a(\cdot)$ for all $a \in \mathcal{S}_{-c}$. Note that (11), (12) introduce the dual-space operator kernel $\widetilde{\mathcal{B}}_{\tau}$ which propagates the dual equivalently to propagation in the original space by $\widetilde{S}_{\tau}$. 
Proposition 3.5. Let $\phi \in \mathcal{S}_{-c}$ with semiconvex dual denoted by $a$. Define $\phi^{1}=\widetilde{S}_{\tau}[\phi]$. Then $\phi^{1} \in \mathcal{S}_{-(c+\eta \tau)}$, and $\phi^{1}(x)=\psi(x, \cdot) \odot a^{1}(\cdot)$ where $a^{1}(x)=\widetilde{\mathcal{B}}_{\tau}(x, \cdot) \odot a(\cdot)$.

Theorem 3.6. Let $V \in \mathcal{S}_{-c}$, and let $a$ be its semiconvex dual (with respect to $\psi$ ). Then $V=$ $\widetilde{S}_{\tau}[V]$ if and only if

$$
\begin{aligned}
a(z) & =\int_{\mathbb{R}^{n}}^{\oplus} \widetilde{\mathcal{B}}_{\tau}(z, y) \otimes a(y) d y \\
& =\widetilde{\mathcal{B}}_{\tau}(z, \cdot) \odot a(\cdot)=\widehat{\widetilde{\mathcal{B}}}_{\tau}[a](z) \quad \forall z \in \mathbb{R}^{n} .
\end{aligned}
$$

Corollary 3.7. The value function $\widetilde{V}$ is given by $\widetilde{V}(x)=\psi(x, \cdot) \odot \widetilde{a}(\cdot)$ where $\widetilde{a}$ is the unique solution of $\widetilde{a}(y)=\widetilde{\mathcal{B}}_{\tau}(y, \cdot) \odot \widetilde{a}(\cdot) \forall y \in \mathbb{R}^{n}$ or equivalently, $\widetilde{a}=\widehat{\widetilde{\mathcal{B}}}_{\tau}[\widetilde{a}]$.

Similarly, for each $m \in \mathcal{M}$ and $z \in \mathbb{R}^{n}$, $S_{\tau}^{m}[\psi(\cdot, z)] \in \mathcal{S}_{-(c+\eta \tau)}$ and

$$
S_{\tau}^{m}[\psi(\cdot, z)](x)=\psi(x, \cdot) \odot \mathcal{B}_{\tau}^{m}(\cdot, z) \quad \forall x \in \mathbb{R}^{n}
$$

where

$$
\mathcal{B}_{\tau}^{m}(y, z)=\left\{\psi(\cdot, y) \odot\left[S_{\tau}^{m}[\psi(\cdot, z)]\right]^{-}(\cdot)\right\}^{-} .
$$

As before, it will be handy to define the max-plus linear operator with "kernel" $\mathcal{B}_{\tau}^{m}$ as $\widehat{\mathcal{B}}_{\tau}^{m}[a](z) \doteq$ $\mathcal{B}_{\tau}^{m}(z, \cdot) \odot a(\cdot)$ for all $a \in \mathcal{S}_{-c}$. Further, one also obtains analogous results (by similar proofs). In particular, one has the following

Theorem 3.8. Let $V \in \mathcal{S}_{-c}$, and let $a$ be its semiconvex dual (with respect to $\psi$ ). Then $V=$ $S_{\tau}^{m}[V]$ if and only if $a(z)=\mathcal{B}_{\tau}^{m}(z, \cdot) \odot a(\cdot) \forall z \in$ $\mathbb{R}^{n}$.

Corollary 3.9. Each value function $V^{m}$ is given by $V^{m}(x)=\psi(x, \cdot) \odot a^{m}(\cdot)$ where each $a^{m}$ is the unique solution of $a^{m}(y)=\mathcal{B}_{\tau}^{m}(y, \cdot) \odot a^{m}(\cdot)$ $\forall y \in \mathbb{R}^{n}$.

\section{DISCRETE TIME APPROXIMATION}

The method developed here will not involve any discretization over space nor any basis functions. Of course this is obvious since otherwise one could not avoid the curse-of-dimensionality. The discretization will be over time, where approximate $\mu$ processes will be constant over the length of each time-step.

We define the operator $\bar{S}_{\tau}$ on $\mathcal{G}_{\delta}$ by

$$
\bar{S}_{\tau}[\phi](x)=\sup _{w \in \mathcal{W}} \max _{m \in \mathcal{M}}\left[\int_{0}^{\tau} l^{m}\left(\xi_{t}^{m}\right)-\frac{\gamma^{2}}{2}\left|w_{t}\right|^{2} d t\right.
$$

$$
\begin{aligned}
& \left.+\phi\left(\xi_{\tau}^{m}\right)\right](x) \\
& =\max _{m \in \mathcal{M}} S_{\tau}^{m}[\phi](x)
\end{aligned}
$$

where $\xi^{m}$ satisfies (2). Let

$$
\overline{\mathcal{B}}_{\tau}(y, z) \doteq \max _{m \in \mathcal{M}} \mathcal{B}_{\tau}^{m}(y, z)=\bigoplus_{m \in \mathcal{M}} \mathcal{B}_{\tau}^{m}(y, z) .
$$

The corresponding max-plus linear operator is

$$
\widehat{\overline{\mathcal{B}}}_{\tau}=\bigoplus_{m \in \mathcal{M}} \widehat{\mathcal{B}}_{\tau}^{m}
$$

Lemma 4.1. For all $z \in \mathbb{R}^{n}, \bar{S}_{\tau}[\psi(\cdot, z)] \in$ $\mathcal{S}_{-(c+\eta \tau)}$. Further, $\bar{S}_{\tau}[\psi(\cdot, z)](x)=\psi(x, \cdot) \odot$ $\overline{\mathcal{B}}_{\tau}(\cdot, z)$.

One has $S_{\tau}^{m} \leq \bar{S}_{\tau} \leq \widetilde{S}_{\tau}$ for all $m \in \mathcal{M}$. With $\tau$ acting as a time-discretization step-size, let

$$
\begin{gathered}
\mathcal{D}_{\infty}^{\tau}=\{\mu:[0, \infty) \rightarrow \mathcal{M} \mid \text { for each } n \in \mathbf{N} \cup\{0\}, \\
\text { there exists } m_{n} \in \mathcal{M} \text { such that } \\
\left.\mu(t)=m_{n} \forall t \in[n \tau,(n+1) \tau)\right\},
\end{gathered}
$$

and for $T=\bar{n} \tau$ with $\bar{n} \in \mathbf{N}$ define $\mathcal{D}_{T}^{\tau}$ similarly but with domain $[0, T)$ rather than $[0, \infty)$. Let $\mathcal{M}^{\bar{n}}$ denote the outer product of $\mathcal{M}, \bar{n}$ times. Let $T=\bar{n} \tau$, and define

$$
\overline{\bar{S}}_{T}^{\tau}[\phi](x)=\max _{\left\{m_{k}\right\}_{k=0}^{\bar{n}-1} \in \mathcal{M}^{\bar{n}}}\left\{\prod_{k=0}^{\bar{n}-1} S_{\tau}^{m_{k}}\right\}[\phi](x)
$$

where the $\prod$ indicates operator composition.

We will be approximating $\tilde{V}$ by solving $V=\bar{S}_{\tau}[V]$ via its dual problem $a=\widehat{\overline{\mathcal{B}}}_{\tau}[a]$ for small $\tau$. Consequently, we will need to show that there exists a solution to $V=\bar{S}_{\tau}[V]$, that the solution is unique, and that it can be found by solving the dual problem. We begin with existence.

Theorem 4.2. Let

$$
\bar{V}(x) \doteq \lim _{N \rightarrow \infty} \overline{\bar{S}}_{N \tau}^{\tau}[0](x)
$$

for all $x \in \mathbb{R}^{n}$ where 0 here represents the zerofunction. Then, $\bar{V}$ satisfies

$$
V=\bar{S}_{\tau}[V], \quad V(0)=0 .
$$

Further, $0 \leq V^{m} \leq \bar{V} \leq \widetilde{V}$ for all $m \in \mathcal{M}$, and consequently, $\bar{V} \in \mathcal{G}_{\delta}$.

Similar techniques to those used for $V^{m}$ and $\widetilde{V}$ will prove uniqueness for (14) within $\mathcal{G}_{\delta}$.

Theorem 4.3. $\bar{V}$ is the unique solution of (14) within the class $\mathcal{G}_{\delta}$ for sufficiently small $\delta>0$. Further, given any $V \in \mathcal{G}_{\delta}, \lim _{N \rightarrow \infty} \overline{\bar{S}}_{N \tau}^{\tau}[V](x)=$ $\bar{V}(x)$ for all $x \in \mathbb{R}^{n}$ (uniformly on compact sets). 
Henceforth, we let $\delta>0$ be sufficiently small such that $V^{m}, \widetilde{V}, \bar{V} \in \mathcal{G}_{\delta}$ for all $m \in \mathcal{M}$.

Theorem 4.4. Let $V \in \mathcal{S}_{-c}$, and let $a$ be its semiconvex dual. Then $V=\bar{S}_{\tau}[V]$ if and only if $a(y)=\overline{\mathcal{B}}_{\tau}(y, \cdot) \odot a(\odot) \forall y \in \mathbb{R}^{n}$.

Corollary 4.5. Value function $\bar{V}$ given by (13) is in $\mathcal{S}_{-c}$, and has representation $\bar{V}(x)=\psi(x, \cdot) \odot$ $\bar{a}(\cdot)$ where $\bar{a}$ is the unique solution of $\bar{a}=\widehat{\overline{\mathcal{B}}}_{\tau}[\bar{a}]$.

The following result on propagation of the semiconvex dual will also come in handy.

Proposition 4.6. Let $\phi \in \mathcal{S}_{-c}$ with semiconvex dual denoted by $a$. Define $\phi^{1}=\bar{S}_{\tau}[\phi]$. Then $\phi^{1} \in \mathcal{S}_{-(c+\eta \tau)}$, and $\phi^{1}(x)=\psi(x, \cdot) \odot a^{1}(\cdot)$ where $a^{1}(y)=\overline{\mathcal{B}}_{\tau}(y, \cdot) \odot a(\cdot) \forall y \in \mathbb{R}^{n}$.

The next result indicates that one may approximate $\widetilde{V}$, the solution of $V=\widetilde{S}_{\tau}[V]$, to as accurate a level as one desires by solving $V=\bar{S}_{\tau}[V]$ for sufficiently small $\tau$. Recall that if $V=\bar{S}_{\tau}[V]$, then it satisfies $V=\overline{\bar{S}}_{N \tau}^{\tau}[V]$ for all $N>0$ (while $\widetilde{V}$ satisfies $V=\widetilde{S}_{N \tau}[V]$ ), and so this is essentially equivalent to introducing a discrete-time $\bar{\mu} \in \mathcal{D}_{N \tau}^{\tau}$ approximation to the $\mu$ process in $\widetilde{S}_{N \tau}$.

Theorem 4.7. Given $\bar{\varepsilon}>0$ and $R<\infty$, there exists $\tau>0$ such that

$$
\widetilde{V}(x)-\bar{\varepsilon} \leq \bar{V}(x) \leq \widetilde{V}(x) \quad \forall x \in \bar{B}_{R}(0) .
$$

\section{ALGORITHM AND EXAMPLES}

Due to space limitations, we cannot give the steps in the actual algorithm that is generated by the above theory. However, we note that at each time step, one generates a set of quadratic functions, where the coefficients in these functions are obtained purely analytically. The approximate solution can be obtained at any point by taking the maximum of these quadratic functions. In the absence of any pruning techniques, the number of quadratic functions at iteration $N$ grows like $M^{N}$. For large $N$, this is indeed a large number. There exist means (such as pruning) for reducing this growth, but we do not discuss them here. Nevertheless, for small values of $M$, we obtain a very rapid solution of such nonlinear HJB PDEs, as will be indicated in the example to follow. Further, the computational cost growth in space dimension $n$ is limited to cubic growth. We emphasize that the existence of an algorithm avoiding the curseof-dimensionality is significant regardless of the practical issues.

A number of examples have so far been tested. In these tests, the computational speeds were very great. (Again, some practical issues involving pruning and initialization are not discussed here due to space limitations.) This is due to the fact that $M=\# \mathcal{M}$ was small. The algorithm as described above was coded in MATLAB (with a very simple pruning technique and initialization). The quoted computational times were obtained with a standard 2001 PC. The times correspond to the time to compute $\bar{V}_{N} \doteq \overline{\bar{S}}_{N \tau}^{\tau}[0]$. The plots below require one to compute the value function and/or gradients pointwise on planes in the state space. These plotting computations are not included in the quoted computational times.

Consider a four-dimensional example with constituent Hamiltonians, $H^{m}$, whose $A^{m}$ are

$$
\begin{aligned}
A^{1} & =\left[\begin{array}{cccc}
-1.0 & 0.5 & 0.0 & 0.1 \\
0.1 & -1.0 & 0.2 & 0.0 \\
0.2 & 0.0 & -1.5 & 0.1 \\
0.0 & -0.1 & 0.0 & -1.5
\end{array}\right], \\
A^{2} & =\left(A^{1}\right)^{T}, \\
A^{3} & =\left[\begin{array}{cccc}
-1.0 & 0.5 & 0.0 & 0.1 \\
0.1 & -1.0 & 0.2 & 0.0 \\
0.2 & 0.0 & -1.6 & -0.1 \\
0.0 & -0.05 & 0.1 & -1.5
\end{array}\right] .
\end{aligned}
$$

The $D^{m}$ and $\Sigma^{m}$ were simply

$$
D^{1}=D^{2}=D^{3}=\left[\begin{array}{llll}
1.5 & 0.2 & 0.1 & 0.0 \\
0.2 & 1.5 & 0.0 & 0.1 \\
0.1 & 0.0 & 1.5 & 0.0 \\
0.0 & 0.1 & 0.0 & 1.5
\end{array}\right]
$$

and

$$
\Sigma^{1}=\Sigma^{2}=\Sigma^{3}=\left[\begin{array}{cccc}
0.2 & -0.01 & 0.02 & 0.01 \\
-0.01 & 0.2 & 0.0 & 0.0 \\
0.02 & 0.0 & 0.25 & 0.0 \\
0.01 & 0.0 & 0.0 & 0.25
\end{array}\right]
$$

The results of this four-dimensional example appear in Figures 1-4. In this case, the results have been plotted over the region of the affine plane $x_{3}=3, x_{4}=-0.5$ given by $x_{1} \in[-10,10]$ and $x_{2} \in[-10,10]$. The backsubstitution error has been scaled by dividing by $|x|^{2}+10^{-5}$. The computations required approximately 40 seconds.

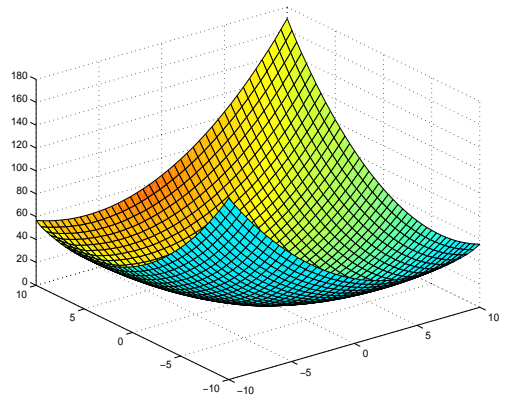

Fig. 1. Value function (4-D case) 


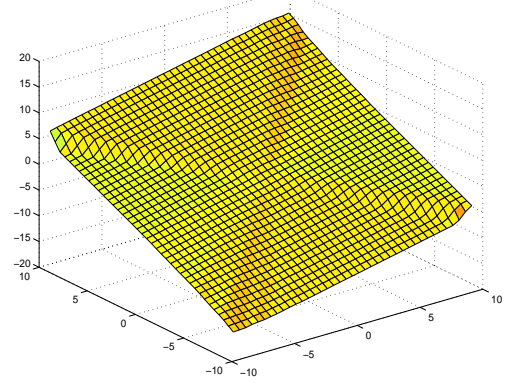

Fig. 2. Partial with respect to $x_{1}$ (4-D case)

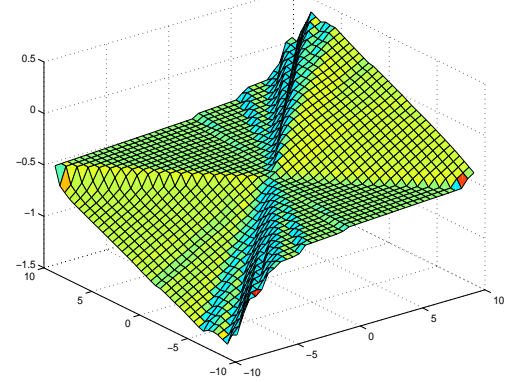

Fig. 3. Partial with respect to $x_{4}$ (4-D case)

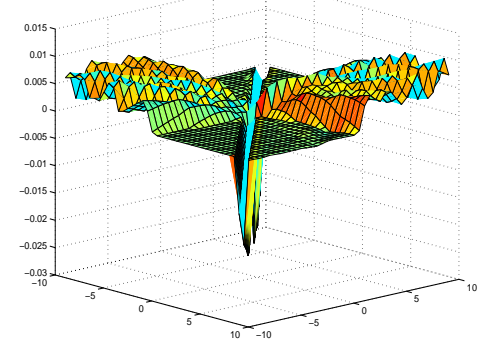

Fig. 4. Scaled backsubstitution error (4-D case)

In order to indicate that the form of HJB PDE solutions obtained by this approach are not limited to the type of shapes appearing in the previous example, we include an additional partial derivative plot from another example, and this is depicted in Figure 5. There is not space here to give the full details of the example, but we note that this example includes constant and linear terms in the $H^{m}$ so as to yield a system where the behavior changes when the state exceeds a certain bound.

\section{REFERENCES}

Akian, M., S. Gaubert and A. Lakhoua (2004), A max-plus finite element method for solving finite horizon determinsitic optimal control problems, Proc. MTNS 2004.

Baccelli, F.L., G. Cohen, G.J. Olsder and J.-P. Quadrat (1992), Synchronization and Linearity, John Wiley.

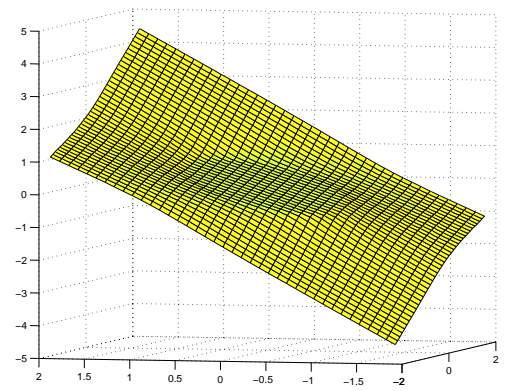

Fig. 5. Partial with respect to $x_{1}$, extra example

Bardi, M. and I. Capuzzo-Dolcetta (1997), Optimal Control and Viscosity Solutions of Hamilton-Jacobi-Bellman Equations, Birkhauser.

Cohen, G., S. Gaubert and J.-P. Quadrat (2004), Duality of Idempotent Semimodules, Linear Algebra and Applications, (to appear).

Dupuis, P., and M. Boué (1999), Markov chain approximations for deterministic control problems with affine dynamics and quadratic cost in the control, SIAM J. on Numerical Analysis, 36, pp. 667-695.

Fleming, W.H. and W.M. McEneaney (2000), A max-plus based algorithm for an HJB equation of nonlinear filtering, SIAM J. Control and Optim., 38, pp. 683-710.

Kolokoltsov, V.N. and V.P. Maslov (1997), Idempotent Analysis and Its Applications, Kluwer.

Litvinov, G.L., V.P. Maslov and G.B. Shpiz (2001), Idempotent Functional Analysis: An Algebraic Approach, Mathematical Notes, 69, 5, 696-729.

Maslov, V.P. (1987), On a new principle of superposition for optimization problems, Russian Math. Surveys, 42, 43-54.

McEneaney, W.M. (2004), Max-Plus Eigenvector Representations for Solution of Nonlinear $\mathrm{H}_{\infty}$ Problems: Error Analysis, SIAM J. Control and Optim. 43, 379-412.

McEneaney, W.M. (2003), Max-Plus Eigenvector Representations for Solution of Nonlinear $\mathrm{H}_{\infty}$ Problems: Basic Concepts, IEEE Trans. Auto. Control, 48, 1150-1163.

McEneaney, W.M. (1998), A Uniqueness result for the Isaacs equation corresponding to nonlinear $\mathrm{H}_{\infty}$ control, Math. Controls, Signals and Systems, 11, 303-334.

Rockafellar, R.T. and R.J. Wets (1997), Variational Analysis, Springer-Verlag.

J. Rust (1997), Using randomization to break the curse of dimensionality, Econometrica, 65, 487-516.

Soravia, P., (1996), $\mathrm{H}_{\infty}$ control of nonlinear systems: differential games and viscosity solutions, SIAM J. Control and Optim., 10711097. 